\title{
Signals Which Build a Tubule
}

\author{
Anil Karihaloob $^{b}$ Christian Nickel $^{a}$ Lloyd G. Cantley ${ }^{b}$ \\ aDepartment of Nephrology, University of Freiburg, Freiburg, Germany; bection of Nephrology, \\ Yale University School of Medicine, New Haven, Conn., USA
}

\author{
Key Words \\ Tubulogenesis · Kidney $\cdot$ MAPK · Signaling $\cdot$ Growth \\ factor
}

\begin{abstract}
The phenomenon of branching morphogenesis is a fundamental process critical for development of several tubular organs including lung, mammary gland, and kidney. In the case of kidney, the ureteric bud (UB) that extends out from a pre-existing epithelial tube, the Wolffian duct, gives rise to the branched collecting duct system while the surrounding metanephric mesenchyme undergoes mesenchymal-epithelial transition to form the proximal parts of the nephron. These events are mediated by several soluble factors that act in a cooperative fashion either as pro or anti tubulogenic factors. Among the growing list of such molecules are the members of the FGF, TGF- $\beta$, and Wnt families as well as GDNF, HGF, and EGF. Cells respond to these soluble factors by initiating signaling pathways that regulate cell proliferation, cell migration and cell morphogenesis. These signaling pathways are also regulated in parallel by cell-cell and cell-matrix interactions, leading to the complex events necessary for tubule formation. Recent in-vitro and in-vivo studies have begun to shed light on the overall regulation of this phenomenon while the specific subcellular mechanisms are only beginning to be
\end{abstract}

understood. This review focuses on our understanding of the morphogenic responses that regulate in-vitro tubulogenesis and how they may help us to ultimately understand this process in vivo in the kidney.

Copyright $@ 2005$ S. Karger AG, Basel

\section{Introduction}

During development of the mammalian kidney, two distinct processes control nephron formation, branching of the ureteric bud tips to create the collecting system of the kidney, and mesenchymal-to-epithelial transformation of the metanephric mesenchyme at the terminal branches of the ureteric bud followed by tubular organization and elongation to form the proximal segments of the nephron. Repair of the injured adult kidney appears to involve a similar process of cellular differentiation and organization into a functional tubular epithelium. These cells are derived either from surviving de-differentiated tubular epithelial cells, or from an influx of more primitive stem cells (from the renal interstitium and/or external sources such as bone marrow), or both. Studying the process of tubule formation in vitro can provide us with valuable information regarding the way in which secreted growth factors, cell-cell interactions, and cell-matrix interactions can regulate this process of mesenchymal-to-epithelial transition and tubular organization.

\section{KARGER \\ Fax +4161306 1234 \\ E-Mail karger@karger.ch \\ www.karger.com

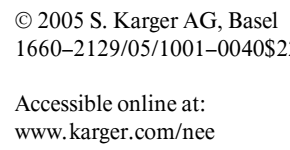

Anil Karihaloo

Section of Nephrology, Yale University School of Medicine

333 Cedar St., LMP 2073

New Haven, CT 06520 (USA)

Tel. +1 203785 7111, Fax +1 203785 4904, E-Mail Anil.Karihaloo@yale.edu 


\section{Intracellular Signaling during Tubule Formation}

In 1991, Montesano et al. [20] demonstrated that hepatocyte growth factor (HGF) could induce Madine-Darby canine kidney (MDCK) cell-derived cysts to form tubular structures when cultured suspended in a collagen matrix. Since then, several other growth factors have been found to modulate tubulogenesis in specific epithelial cell types. These include positive stimulators such as the EGF receptor ligands, epidermal growth factor (EGF) and transforming growth factor- $\alpha$ (TGF- $\alpha$ ) and negative regulators such as TGF- $\beta$. EGF and EGFR are expressed in embryonic kidney and some genetic backgrounds EGFR knockout mice reveal a defective collecting duct system $[32,34]$. In contrast, mice lacking HGF or its receptor $c$ met do not show any abnormal kidney development. However, since those mice die by embryonic day 14 , one cannot exclude the possibility that HGF may be involved in the later stages of kidney tubule development. Among the most prominent negative regulators is TGF- $\beta$. TGF- $\beta$ was shown to primarily inhibit HGF induced tubule branching but not the elongation of the tubule [31]. Other TGF- $\beta$ family members such as bone morphogenetic proteins (BMP) can also modulate in-vitro tubulogenesis in mouse renal collecting duct cells. BMP2 and high doses of BMP7 markedly inhibit tubule formation, while low doses of BMP7 actually stimulate tubulogenesis [6]. Interestingly, the glial cell derived neurotrophic factor, GDNF, a growth factor that is essential for the budding of the UB from the caudal Wolffian duct [21,37], does not induce branching in isolated UB cells [30].

The cellular events triggered by tubulogenic growth factor/receptor combinations such as HGF and EGF are delegated by a complex series of cytosolic and membrane associated signaling events that we are just beginning to understand. These signaling steps modulate cell shape change, cell division and cell motility, and are integral components of the formation of tubules both in vitro and in vivo. Following growth factor stimulation, tyrosine kinase receptors homodimerize resulting in phosphorylation of tyrosine residues. These residues in turn act as binding sites for various proteins containing src-homology 2 ( $\mathrm{SH} 2$ ) domains. In the case of HGF receptor, c-met, some of these signaling pathways are directly activated (MAPK pathway via binding of the Grb2-Sos complex to c-met, for example), some are indirectly activated by binding to intermediate docking proteins such as Gab1 (SHP2 for example), while others can be activated by both direct and indirect mechanisms (phospholipase $\mathrm{C} \gamma$ (PLC $\gamma$ ) and phosphoinositide 3-kinase (PI3-kinase), for detailed review see [40]).
Disrupting the association of these $\mathrm{SH} 2$-domain containing proteins with the signaling complex by sitedirected mutagenesis leads to either complete or partial inhibition of both cell migration and tubulogenesis [38]. Similarly, blocking specific signaling cascades with chemical inhibitors such as wortmanin (PI3-K inhibitor) or UO126 (MAPK pathway inhibitor) can disrupt the migratory and tubulogenic responses induced by either HGF or EGF. Interestingly however, EGF requires activation of the ERK5 pathway (also called big MAP kinase, BMK) for its tubulogenic effects in IMCD cells, whereas HGF primarily utilizes activation of ERK1/2 [14].

Recent studies have shown that it is not only which signaling pathways are activated but also the degree; duration and the localization of activation are also critical. For example, both a transient high level activation and a more sustained low level activation of the ERK1/2 as well as PI3-K pathways are required for HGF induced tubulogenesis [7, 13,35], suggesting a potential role for both signaling at the membrane and transcriptional regulation. Indeed, sustained ERK activation is important for induction of ETS-1 transcription factor that is activated in organs undergoing branching morphogenesis [9] while a high level ERK activation at the membrane is required for focal adhesion turnover and cell migration [10].

In addition to the aforementioned transducers, p38 MAPK, which is highly expressed during kidney development [25], has also been shown to play a role in tubulogenesis. The p38 MAPK inhibitor, SB203580, inhibits growth of rat embryonic metanephroi without any significant effect on ureteric bud branching [8]. However, low doses of BMP7 have been shown to induce branching morphogenesis by activating p38 MAPK [9], suggesting that different mechanisms may exist for p38 MAPK dependent morphogenic effects. In addition, p38 and ERK have been found to be significantly activated in dysplastic epithelia from human renal dysplasia, suggesting that they may play a role in cyst formation/proliferation [24].

In addition to cell proliferation, tubule growth requires a regulated change in cell shape, necessitating reorganization of the actin cytoskeleton. This process has been shown to be regulated by the Rho family of small GTPases (Rho, Rac and cdc42) that can be activated downstream of the PI 3-K and ras. These events lead to actin polymerization at the cell periphery resulting in the extension of lamellipodia (that are essential for cell migration) and filopodia (that precede de novo in vitro tubulogenesis). In contrast, RhoA acting via it downstream effector ROCK (Rho-associated kinase), stimulates myosin light chain phosphorylation and regulates actin stress 
fiber formation and cell contractility. Thus, a coordinated activation and deactivation of Rac and Rho is required for cell shape change and migration.

\section{Cellular Events during Tube Formation in vitro}

When renal tubular epithelial cells are isolated and grown in culture, they spontaneously form and maintain cell-cell contacts. In 2-dimensional culture (on Transwell filters for example), these cell-cell contacts promote tight junction formation and vectorial transport. When these same cells are cultured suspended in a 3-dimensional matrix, they organize to give rise to either multicellular cysts or elongated tubes that can form lumens, thus mimicking a tubule. How do these cells undergo the specific shape changes and organization that leads to the generation of tubes? Five general mechanisms have been described to date, wrapping (as in neural tube development), cavitation (in which a mass of cells forms a lumen by apoptosis of the central cells, as in salivary gland formation), cell hollowing (where a single cell forms a membrane bound cavity), budding, and cord hollowing [17]. In budding, as the name suggests, cells extend out and away from the epithelial sheet, forming a tube as the bud elongates (as is seen in ureteric bud outgrowth from the Wolffian duct during kidney development). The resultant tube may undergo further terminal dichotomous branching (as in the kidney) or a new branch may extend from the side of an existing branch/tube (as during development of respiratory system). Cord hollowing is seen when a linear array of cells polarizes to delineate an apical membrane with a central lumen. The major difference between budding and cord hollowing is the state of polarization of the pre-existing epithelia. Budding occurs from an already polarized cyst or tube of epithelia, whereas cord hollowing is coincident with polarization/lumen formation.

In vitro cell culture models of tubule formation have provided significant mechanistic insights into the processes involved in tubule formation and repair in vivo. Using MDCK and IMCD cells, tubules can form by 2 distinct, but overlapping, processes; budding from an existing cyst or de novo cord formation from individual cells. Budding appears to recapitulate the initial events described in ureteric bud formation (although a central lumen is always maintained in the ureteric bud but not in cyst budding), whereas cord hollowing may be more representative of the processes regulating comma and Sshaped body formation during development of the proximal nephron.
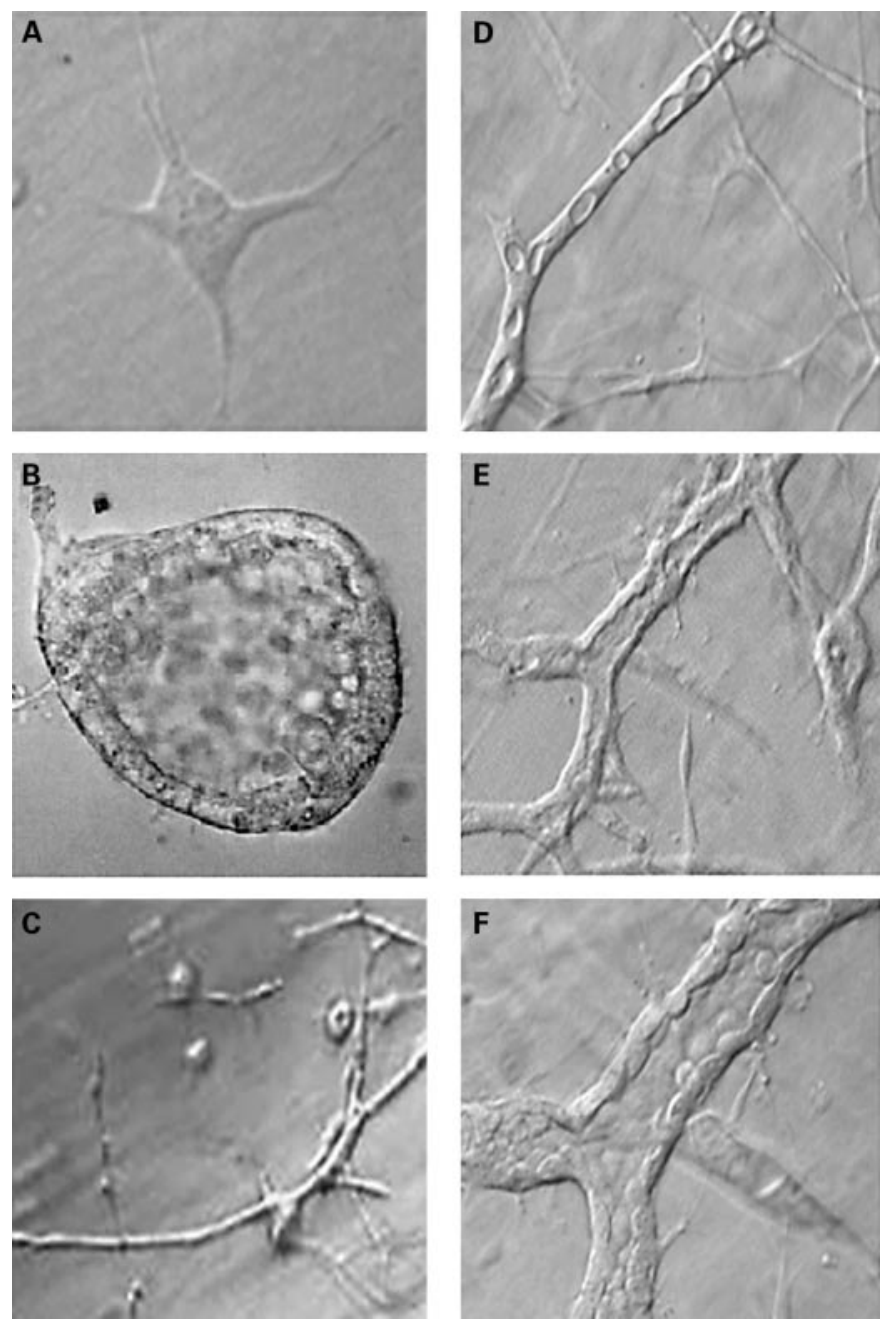

Fig. 1. mIMCD-3 cells were grown in 3D matrix of either collagen, in the presence of $40 \mathrm{ng} / \mathrm{ml}$ of $\mathrm{HGF}(\mathbf{A})$, or pure Matrigel (B) or a mixture of collagen and Matrigel + HGF, for 1-6 days. Cells suspended in Matrigel form multi-cellular cysts with central lumen whereas cells suspended in a collagen and Matrigel mix transit from early single cell branch to multi-cellular cords (C) without any visible lumens. By day 4, multiple vacuoles (D) form within this cord of cells (cord hollowing) that coalesce by day $5(\mathbf{E})$ eventually giving rise to a multicellular tubule with single lumen by day $6-8$. F is the same as $\mathbf{E}$ but at a higher magnification.

\section{Cell-Cell Interaction}

When MDCK or IMCD cells are grown suspended in a collagen gel in the absence of tubulogenic growth factors, they spontaneously form cysts that consist of a monolayer of cells surrounding a fluid filled central lumen (fig. 1B). These cells appear to fully polarize with apical localization of proteins such as gp135, basal localization of matrix 
adhesion proteins, and lateral localization of junctional proteins such as E-cadherin and ZO-1. When HGF is added, some of the cyst cells bud out from the central cavity and migrate into the surrounding matrix, transiently losing their polarity and de-differentiating as has been described following ischemic injury of the proximal tubule. The lumen is lost as the apical protein gp135 and the basolateral protein desmoplakin are redistributed from the plasma membrane to the cytoplasm, and the budding cells elongate, proliferate, and migrate away from the cyst forming a several cell-thick cord. During this de-differentiation process, cell-cell complexes break down leading to re-distribution of cadherins (such as E-cadherin and Kspcadherin) from discreet lateral junctions to more diffuse plasma membrane localization or to the cytoplasm.

Cadherins not only serve to stabilize adherens junctions by linking the cell-cell junction to the actin cytoskeleton, they also serve a signaling function via their interaction with $\beta$-catenin. $\beta$-catenin binds to the cytoplasmic domain of cadherins and is involved in linking the cadherin-catenin complex to the actin cytoskeleton [1]. However, $\beta$-catenin is also a component of the canonical Wnt signaling pathway. Several Wnts are expressed during kidney development, and activation of the Wnt pathway can result in localization of $\beta$-catenin to the nucleus where it activates a pattern of gene expression that is required for normal nephron development. A recent study using MDCK cells indicates that canonical Wnt signaling through $\beta$-catenin contributes to cell growth and survival but may not play a role in morphogenic responses [18]. (For a detailed review on the role of Wnts in kidney development and branching morphogenesis refer to $[27,36]$.) Interestingly, dissociation of cell-cell contacts also results in $\beta$-catenin translocation to the nucleus [22], raising the possibility that this signaling pathway then plays a role in the subsequent re-differentiation of the budding cells to re-form a functional tubule. In support of a role for $\beta$-catenin in this process, deleting the NH2-terminal of $\beta$-catenin results in abolition of HGF-mediated tubulogenesis, even though the cells proliferate normally [29].

When MDCK or IMCD cells are grown in matrix in the presence of HGF from the beginning, they fail to form cysts but instead change to an elongated, spindle-like morphology and extend processes out into the matrix (fig. 1A). When these processes stably contact adjacent cells, cell-cell interactions signal the de novo formation of elongated cords of cells rather than cysts (fig. 1C). One of these cell-cell regulatory interactions occurs via activation of EphA, a member of the Eph family of transmembrane receptor tyrosine kinases. Ligands for Eph kinases are ephrins located on nearby cells. These can be either GPI linked (ephrin-A) or transmembrane proteins (ephrin-B). Both Eph and ephrins can transmit signals to the cell. Recently, Miao et al. [19] demonstrated that activation of EphA kinases in MDCK cells negatively regulate HGF induced branching morphogenesis by suppressing Rac1 without inhibiting RhoA. This might provide a mechanism whereby stabilizing a cell-cell contact would inhibit further attempts at branching, thus favoring the generation of an elongated tubule with fewer branch points.

Regardless of whether the cord of cells forms via dedifferentiation and extension of a bud from an existing cyst or de novo formation of a cord from proliferating cells, when a critical density of cells is achieved, lumen formation occurs by a process of cord hollowing. This begins by the formation of multiple membrane-lined vesicles (fig. 1D), followed by fusion into a single central lumen (fig. 1E). It is relevant to note that the appearance of the lumen appears to coincide with establishment of cell-density dependent apical-basal polarity. Recently, Cohen's group demonstrated that EMK1, the mammalian orthologue of the Caenorhabditis elegans protein Par1, is essential in determining polarity and lumen formation in MDCK cells. EMK1 was shown to promote reorganization of the microtubule network that in turn regulates lumen formation. The expression and localization of EMK1 may indeed be the cue that determines the axis of polarity during tubulogenesis.

\section{Cell-Matrix Interaction}

The importance of cell-matrix interactions in determining the architecture of epithelial structures is demonstrated by the observation that when IMCD cells grown suspended in pure Matrigel they form multicellular cysts whereas in type I collagen they form branching cords. However, when cultured in a mixture of collagen and Matrigel, these cells give rise to elongated, branched tubules with visible lumens (fig. 1F). Studies of these matrix components have demonstrated that laminin and fibronectin promote branching and tubule formation, whereas collagen IV and vitronectin inhibit the process [33].

The major family of cell membrane proteins involved in mediating cell interaction with the extracellular matrix are the integrins, and their importance in tubule formation has been established by studies in which the addition of function-blocking antibodies to integrins can prevent in vitro tubulogenesis [39]. Integrins are transmembrane 
proteins that facilitate cell adhesion by associating (via their extracellular region) with specific basement membrane components such as type IV collagen, laminin, and fibronectin, while on the cytosolic side, they can signal to the cell interior and interact with the actin cytoskeleton via a large cluster of proteins including vinculin, talin, $\alpha-$ actinin, paxillin and focal adhesion kinase (FAK). Collectively these complexes are termed focal adhesions, and their formation, stabilization, and dissolution must be regulated during tubulogenesis. Recent work by our laboratory and others has demonstrated that paxillin plays a central role in this regulatory process. HGF can stimulate the tyrosine phosphorylation of paxillin [16], resulting in the association of paxillin with ERK, a member of the MAPK family of intracellular signaling proteins. This interaction mediates the serine phosphorylation of paxillin and recruitment/activation of FAK, thus stimulating focal adhesion turnover and promoting extension of cell processes and cell migration. Mutational interruption of this pathway completely eliminates HGF-stimulated tubule formation, instead resulting in cyst formation [10; Ishibe, submitted].

In addition to integrins, it has recently been suggested that another transmembrane protein, polycystin-1 (the gene product that is mutated in autosomal-dominant polycystic kidney disease), localizes to focal adhesions, raising the possibility that polycystins could play a role in cell-matrix interactions as well [5]. In accordance with this idea, our laboratory and others have found that overexpression of full-length polycystin-1 or the cytosolic tail induces spontaneous cell migration and branching morphogenesis in renal epithelial cells [3, 23].

Another family of proteins involved in cell-matrix interactions is the proteoglycans (PGs). Several PGs that form integral parts of the renal basement membrane and/ or mesangial matrices include perlecan, decorin, biglycans, agrin, leprecan and collagen type XVIII. In addition, there are cell surface PGs, such as syndecans and glypicans that participate in these interactions. Recent in-vitro studies have suggested a critical role for these proteins in regulating tubulogenesis as well. Inhibition of PG sulfation has been shown to blunt the branching morphogenesis of the isolated ureteric bud [4], while we have found that cell surface glypicans can bind a proteolytic fragment of collagen XVIII, endostatin, and thereby markedly inhibit branching morphogenesis of either isolated cells or the explanted ureteric bud $[12,15]$.

A third group of proteins that are critical for the regulation of cell-matrix interactions are the matrix metalloproteinases (MMPs). Tubule formation in a 3-dimensional matrix involves cell process extension followed by cell multiplication, migration and polarization. This first step of extending a filopodia requires degradation of the surrounding matrix by MMPs. MMPs can degrade a variety of ECM substrates including, but not limited to, fibronectin, proteoglycans and collagens. Inhibiting MMP activity by a broad range inhibitors, or by expression of tissue inhibitors of metalloproteinases (TIMPs), can block growth factor induced tubulogenesis in vitro and regulate ureteric bud branching [2, 28]. Using IMCD cells, Nigam et al. [28] have demonstrated modulation of MMP-2 expression during tubulogenesis, with the increase and decrease of expression correlating with stimulation and inhibition of branching morphogenesis of these cells, respectively. Kadono et al. [11] have shown an increase in a separate protease, membrane type MMP (MT1-MMP), during the course of tubule formation by MDCK cells in a 3-dimensional collagen gel matrix, although this increase was not initiated by growth factor stimulation. In addition to MMPs, serine proteases such as tissue plasminogen activator (t-PA) can modulate branching morphogenesis in vitro [26], although no obvious renal phenotype has been observed in mice lacking either of the two genes.

Thus, it is clear that multiple signaling cues inside and outside of a cell participate in concert to shape a tubule. The in vitro study of this process has led to the identification of many of these regulatory factors, and is now beginning to shape our understanding of how these factors can interact selectively in certain regions of the cell as well as temporally at different points during the process of dedifferentiation and re-differentiation/transdifferentiation. Ultimately, these studies promise to establish a better understanding of why kidney development and repair are not always properly regulated, and to provide us with the necessary tools to promote proper tubule function. 


\section{References}

-1 Aberle H, Butz S, Stappert J, Weissig H, Kemler R, Hoschuetzky H: Assembly of the cadherin-catenin complex in vitro with recombinant proteins. J Cell Sci 1994; 107:3655-3663.

$>2$ Barasch J, Yang J, Qiao J, Tempst P, ErdjumentBromage H, Leung W, Oliver JA: Tissue inhibitor of metalloproteinase-2 stimulates mesenchymal growth and regulates epithelial branching during morphogenesis of the rat metanephros J Clin Invest 1999;103:1299-1307.

-3 Boletta A, Qian F, Onuchic LF, Bhunia AK, Phakdeekitcharoen B, Hanaoka K, Guggino W, Monaco L, Germino GG: Polycystin-1, the gene product of PKD1, induces resistance to apoptosis and spontaneous tubulogenesis in MDCK cells. Mol Cell 2000;6:1267-1273.

$\checkmark 4$ Davies J, Lyon M, Gallagher JD. Garrod D: Sulphated proteoglycan is required for collecting duct growth and branching but not nephron formation during kidney development. Development 1995;121:1507-1517.

$>5$ Geng L, Burrow CR, Li HP, Wilson PD: Modification of the composition of polycystin-1 multiprotein complexes by calcium and tyrosine phosphorylation. Biochim Biophys Acta 2000;1535:21-35.

6 Grisaru S, Cano-Gauci D, Tee J, Filmus J, Rosenblum ND: Glypican-3 modulates BMPand FGF-mediated effects during renal branching morphogenesis. Dev Biol 2001;231:31-46.

7 Gual P, Giordano S, Williams TA, Rocchi S, Van Obberghen E, Comoglio PM: Sustained recruitment of phospholipase $\mathrm{C}$-gamma to Gabl is required for HGF-induced branching tubulogenesis. Oncogene 2000;19:1509-1518.

$\checkmark 8$ Hida M, Omori S, Awazu M: ERK and p38 MAP kinase are required for rat renal development. Kidney Int 2002;61:1252-1262.

-9 Hu MC, Wasserman D, Hartwig S, Rosenblum ND: p38MAPK acts in the BMP7-dependent stimulatory pathway during epithelial cell morphogenesis and is regulated by Smad1. J Biol Chem 2004;279:12051-12059.

10 Ishibe S, Joly D, Zhu X, Cantley LG: Phosphorylation-dependent paxillin-ERK association mediates hepatocyte growth factor-stimulated epithelial morphogenesis. Mol Cell 2003; 12:1275-1285.

11 Kadono Y, Shibahara K, Namiki M, Watanabe Y, Seiki M, and Sato H: Membrane type 1matrix metalloproteinase is involved in the formation of hepatocyte growth factor/scatter factor-induced branching tubules in Madin-Darby canine kidney epithelial cells. Biochem Biophys Res Commun 1998;251:681-687.

-12 Karihaloo A, Karumanchi SA, Barasch J, Jha V, Nickel CH, Yang J, Grisaru S, Bush KT, Nigam S, Rosenblum ND, Sukhatme VP, Cantley LG: Endostatin regulates branching morphogenesis of renal epithelial cells and ureteric bud. Proc Natl Acad Sci USA 2001;98: 12509-12514.

$\checkmark 13$ Karihaloo A, Kale S, Rosenblum ND, Cantley LG: Hepatocyte growth factor-mediated renal epithelial branching morphogenesis is regulated by glypican- 4 expression. Mol Cell Biol 2004;24:8745-8752.
14 Karihaloo A, O'Rourke DA, Nickel C, Spokes K, Cantley LG: Differential MAPK pathways utilized for HGF- and EGF-dependent renal epithelial morphogenesis. J Biol Chem 2001; 276:9166-9173.

15 Karumanchi SA, Jha V, Ramchandran R, Karihaloo A, Tsiokas L, Chan B, Dhanabal M, Hanai JI, Venkataraman G, Shriver Z, Keiser N, Kalluri R, Zeng H, Mukhopadhyay D, Chen RL, Lander AD, Hagihara K, Yamaguchi Y, Sasisekharan R, Cantley L, Sukhatme VP: Cell surface glypicans are low-affinity endostatin receptors. Mol Cell 2001;7:811-822.

16 Liu ZX, Yu CF, Nickel C, Thomas S, Cantley LG: Hepatocyte growth factor induces ERKdependent paxillin phosphorylation and regulates paxillin-focal adhesion kinase association. J Biol Chem 2002;277:10452-10458.

17 Lubarsky B, Krasnow MA: Tube morphogenesis: making and shaping biological tubes. Cell 2003;112:19-28.

18 Lyons JP, Mueller UW, Ji H, Everett C, Fang X, Hsieh JC, Barth AM, McCrea PD: Wnt-4 activates the canonical beta-catenin-mediated Wnt pathway and binds Frizzled-6 CRD: functional implications of Wnt/beta-catenin activity in kidney epithelial cells. Exp Cell Res 2004; 298:369-387.

19 Miao H, Nickel CH, Cantley LG, Bruggeman LA, Bennardo LN, Wang B: EphA kinase activation regulates $\mathrm{HGF}$-induced epithelial branching morphogenesis. J Cell Biol 2003; 162:1281-1292.

20 Montesano R, Matsumoto K, Nakamura T, Orci L: Identification of a fibroblast-derived epithelial morphogen as hepatocyte growth factor. Cell 1991;67:901-908.

21 Moore MW, Klein RD, Farinas I, Sauer H, Armanini M, Phillips H, Reichardt LF, Ryan AM, Carver-Moore K, Rosenthal A: Renal and neuronal abnormalities in mice lacking GDNF. Nature 1996;382:76-79.

22 Nelson WJ, Nusse R: Convergence of Wnt, beta-catenin, and cadherin pathways. Science 2004;303:1483-1487.

23 Nickel C, Benzing T, Sellin L, Gerke P, Karihaloo A, Liu ZX, Cantley LG, Walz G: The polycystin-1 C-terminal fragment triggers branching morphogenesis and migration of tubular kidney epithelial cells. J Clin Invest 2002;109: 481-489.

24 Omori S, Fukuzawa R, Hida M, Awazu M: Expression of mitogen-activated protein kinases in human renal dysplasia. Kidney Int 2002;61:899-906.

25 Omori S, Hida M, Ishikura K, Kuramochi S, Awazu M: Expression of mitogen-activated protein kinase family in rat renal development. Kidney Int 2000;58:27-37.

26 Pepper MS, Matsumoto K, Nakamura T, Orci L, Montesano R: Hepatocyte growth factor increases urokinase-type plasminogen activator (u-PA) and u-PA receptor expression in MadinDarby canine kidney epithelial cells. J Biol Chem 1992;267:20493-20496.
27 Perantoni AO: Renal development: perspectives on a Wnt-dependent process. Semin Cell Dev Biol 2003; 14:201-208.

28 Pohl M, Sakurai H, Bush KT, Nigam SK: Matrix metalloproteinases and their inhibitors regulate in vitro ureteric bud branching morphogenesis. Am J Physiol Renal Physiol 2000;279: F891-F900.

29 Pollack AL, Barth AI, Altschuler Y, Nelson WJ, Mostov KE: Dynamics of beta-catenin interactions with APC protein regulate epithelial tubulogenesis. J Cell Biol 1997;137:16511662.

30 Qiao J, Sakurai H, Nigam SK: Branching morphogenesis independent of mesenchymal-epithelial contact in the developing kidney. Proc Natl Acad Sci USA 1999;96:7330-7335.

31 Sakurai H, Nigam SK: Transforming growth factor-beta selectively inhibits branching morphogenesis but not tubulogenesis. Am J Physiol 1997;272:F139-F146.

32 Sakurai H, Tsukamoto T, Kjelsberg CA, Cantley LG, Nigam SK: EGF receptor ligands are a large fraction of in vitro branching morphogens secreted by embryonic kidney. Am J Physiol 1997;273:F463-F472

33 Santos OF, Nigam SK: HGF-induced tubulogenesis and branching of epithelial cells is modulated by extracellular matrix and TGF-beta. Dev Biol 1993;160:293-302.

34 Threadgill DW, Dlugosz AA, Hansen LA, Tennenbaum T, Lichti U, Yee D, LaMantia C, Mourton T, Herrup K, Harris RC, et al: Targeted disruption of mouse EGF receptor: effect of genetic background on mutant phenotype. Science 1995;269:230-234.

35 Ueland JM, Gwira J, Liu ZX, Cantley LG: The chemokine $\mathrm{KC}$ regulates HGF-stimulated epithelial cell morphogenesis. Am J Physiol Renal Physiol 2004;286:F581-F589.

36 Vainio SJ: Nephrogenesis regulated by Wnt signaling. J Nephrol 2003;16:279-285.

37 Vega QC, Worby CA, Lechner MS, Dixon JE, Dressler GR: Glial cell line-derived neurotropic factor activates the receptor tyrosine kinase RET and promotes kidney morphogenesis Proc Natl Acad Sci USA 1996;93:1065710661.

38 Weidner KM, Sachs M, Riethmacher D, Birchmeier W: Mutation of juxtamembrane tyrosine residue 1001 suppresses loss-of-function mutations of the met receptor in epithelial cells. Proc Natl Acad Sci USA 1995;92:2597-2601.

39 Zent R, Bush KT, Pohl ML, Quaranta V, Koshikawa N, Wang Z, Kreidberg JA, Sakurai H, Stuart RO, Nigam SK: Involvement of laminin binding integrins and laminin-5 in branching morphogenesis of the ureteric bud during kidney development. Dev Biol 2001;238:289302.

40 Zhang YW, Vande Woude GF: HGF/SF-met signaling in the control of branching morphogenesis and invasion. J Cell Biochem 2003;88: 408-417. 